Ann. Génét. Sél. anim., 1978, 10 (4), 507-5I5.

\title{
Un variant clair du Mouflon Corsico-Sarde (Ovis musimon) dû à un allèle au locus B (Brun)
}

B. DENIS(*), J. J. LAUVERGNE(**) et M. THÉRET(*)

\author{
$\left(^{*}\right)$ Service de Zootechnie, \\ École nationale vétérinaive d'Alfort, \\ 94704 Maisons-Alfort Cedex \\ (**) Département de Génétique animale, \\ Centre national de Recherches zootechniques, I.N.R.A., \\ 78350 Jouy-en-Josas
}

\begin{abstract}
Résumé
Un Mouflon Corsico-Sarde mâle de phénotype "sauvage clair ", qui a été utilisé en France dans une série de croisements pour l'étude de la coloration, s'est avéré porteur d'un allèle récessif au locus $B(B r u n)$. Cet allèle modifie la couleur de l'eumélanine qui, au lieu d'être noir de jais, présente une couleur brun-chocolat plus claire, assez proche d'ailleurs de celle de certaines phoéomélanines.
\end{abstract}

\section{I. - Introduction}

Dans un récent article nous avons étudié la formule génétique de la couleur d'un Mouflon mâle en provenance du Muséum national d'Histoire Naturelle de Paris en le croisant avec des brebis de divers génotypes (LAUVERGNE et al., I977).

Ce mouflon mâle, bien que présentant en gros les caractéristiques colorées de l'espèce, c'est-à-dire le patron sauvage (avec le ventre, la région périnéale et l'intérieur des membres blancs, le corps étant fauve), était d'un type clair, les nuances noires généralement décrites en tête, sur les pattes et sur la ligne du dos ayant fait place à des nuances plus claires.

Dans les croisements effectués nous avions, en gros, retrouvé le patron sauvage avec il est vrai quelques variations que nous avions alors négligées afin de ne pas trop compliquer le schéma explicatif. En ajoutant des données recueillies dans l'intervalle et en utilisant un mode d'examen nouveau nous allons essayer, dans le présent article, d'expliquer génétiquement ce variant clair du mouflon que nous avions appelé Mouflon de Corse mais qu'il faut appeler Corsico-Sarde, cf. Bunch et al, (1978). 


\section{II. - Matériel et méthodes}

\section{A. - Position du problème}

Les dessins caractéristiques d'un patron sauvage comme celui du Mouflon Corsico-Sarde où l'eu- et la phoeomélanine sont mêlées à l'intérieur parfois d'un même poil sont đéterminés essentiellement par l'allèle sauvage en Agouti : $A^{+}$ (cf. SeARLE, rg68 pour l'homologie entre Mammifères).

Dans ces conditions un variant clair de ce patron sauvage peut provenir soit d'un mutant en $A$ gouti dont 1'expressivité serait proche de celle de $A^{+}$soit d'un modificateur de l'expressivité de $A^{+}$.

L'hypothèse d'un allèle en $A$ est facilement testable. N'ayant, en principe, introduit qu'un allèle double recessif lors de nos expériences de croisements à Alfort on ne devrait pas observer de ségrégations clair / foncé parmi les descendants de type sauvage.

Si d'aventure cette ségrégation existait, il faudrait alors chercher un modificateur de l'expressivité d'A gouti. On pense tout d'abord aux allèles situés aux principaux loci contrôlant la pigmentation des mammifères, c'est-à-dire $B$ (Brun), $C$ (Albinisme vrai), $D$ (Dilution), $E$ (Extension du noir) et $P$ (Pink-eyed) (cf. SEARLE, déjà cité).

De ces cinq loci il semble possible d'écarter, du moins dans un premier temps, les loci $C, D$ et $P$. En effet les allèles en $C$ ont tendance à réduire d'abord l'intensité de la teinte de la phoeomélanine alors que, chez notre mutant clair, elle ne semble pas affectée. Les allèles en $D$ donnent une dilution qui, elle aussi, ne semble pas se manifester chez les produits que nous avons observés. Quant aux allèles en $P$ ils affectent bien l'eumélanine mais ils entrainent un éclaircissement de la couleur de l'œil que l'on n'a pas non plus constaté.

Il reste comme possibilité un mutant en $E$ du type récessif $(e)$ qui supprimerait toute présence d'eumélanine ou bien un allèle en $B$ qui transformerait l'eumélanine noire en une eumélanine plus claire. Pour distinguer entre ces deux possibilités il faut examiner la nature du pigment qui, chez le variant clair, a remplacé la mélanine habituellement noire.

\section{B. - Les données disponibles}

On a réexaminé les données de ségrégation déjà utilisées précédemment (LAUVERGNE et al., I977) en leur adjoignant de nouvelles données acquises dans l'intervalle, ce afin de déceler une éventuelle ségrégation entre " sauvage " clair et ordinaire.

Sur les animaux encore disponibles on a procédé à des déterminations de la nature des mélanines du pelage. Pour cette distinction entre eumélanine noire ou brun-chocolat et phoeomélanine rouge ou fauve, l'examen biochimique est idéal; malheureusement il est encore rarement pratiqué et il nous a été impossible de le mettre en place. On lui a substitué un examen au moyen d'une échelle colorimétrique spécialement établie à cet effet. 


\section{C. -- Élaboration d'une échelle colorimétrique}

Des études colorimétriques chez les Ruminants ont été commencées par LAUUERGNE (I966) qui utilisait un atlas coloré pour mesurer les nuances du poil des bovins. Des études biochimiques dans la même espèce ont permis d'identifier avec certitude la nature du pigment chez certaines races (MISURACA et al., I974).

Chez la chèvre, les études colorimétriques de LAUVERGNE et HowELL (I978) complètent les déterminations génétiques des types de mélanines. La détermination génétique d'un type de mélanine se base sur des considérations telles que la présence simultanée et bien individualisée de deux pigments chez le même individu: l'un est forcément de l'eumélanine (même s'il n'est pas noir) et l'autre de la phoeomélanine. Il suffit alors de mesurer la couleur de cette eumélanine non noire pour pouvoir l'identifier sur un animal qui la portera d'une manière uniforme et ce, même si la nuance tend, à première vue, à se confondre avec celle de la phoeomélanine rouge.

Pour le mouton, ADALSTEInsson (I970) avait déjà implicitement raisonné de cette manière pour distinguer l'eumélanine brun-chocolat chez le mouton Islandais, sans donner toutefois de mesure colorimétrique.

Pour notre échelle colorimétrique nous avons procédé à des mesures sur les trois espèces de Ruminants : Bovins, Caprins et Ovins. Pour les deux premières espèces nous avons retenu les observations déjà évoquées en leur adjoignant des mesures sur la chèvre du Rove où les phénotypes phoéomélaniques sont plus courants qu'en race Corse.

Pour les Ovins on a élaboré une échelle à partir d'examens faits en races Corse et Solognote pour la mélanine rouge et de prélèvements Islandais pour l'eumélanine brun-chocolat. Les résultats des mesures sont rassemblés dans le tableau r.

Rappelons que trois paramètres sont nécessaires pour déterminer physiquement une nuance colorée : on part d'une teinte spectrale pure dont on fait varier la

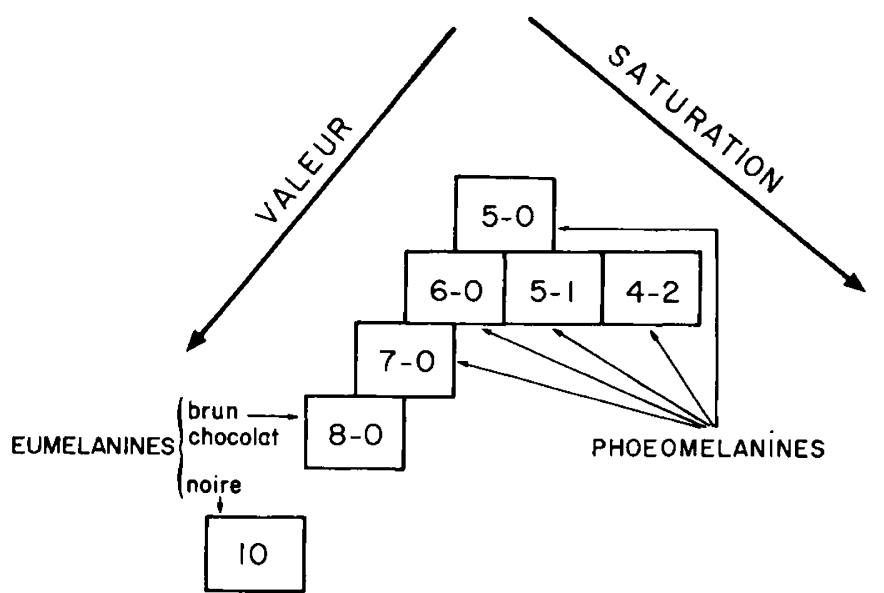

FIG. I. - Localisation dans le plan valeur - saturation des nuances des diverses mélanines des Ruminants; on n'a pas noté les couleurs spectrales qui sont dans les oranges: de 8 à $I 2$ selon l'atlas de MüLLER.

Localisation in the lightness-saturation plane of the shades of various melanins from Ruminants. The hues, which are omitted, are between 8 and I2 according MÜLLER's atlas. 
B. DENIS, J. J. LAUVERGNE

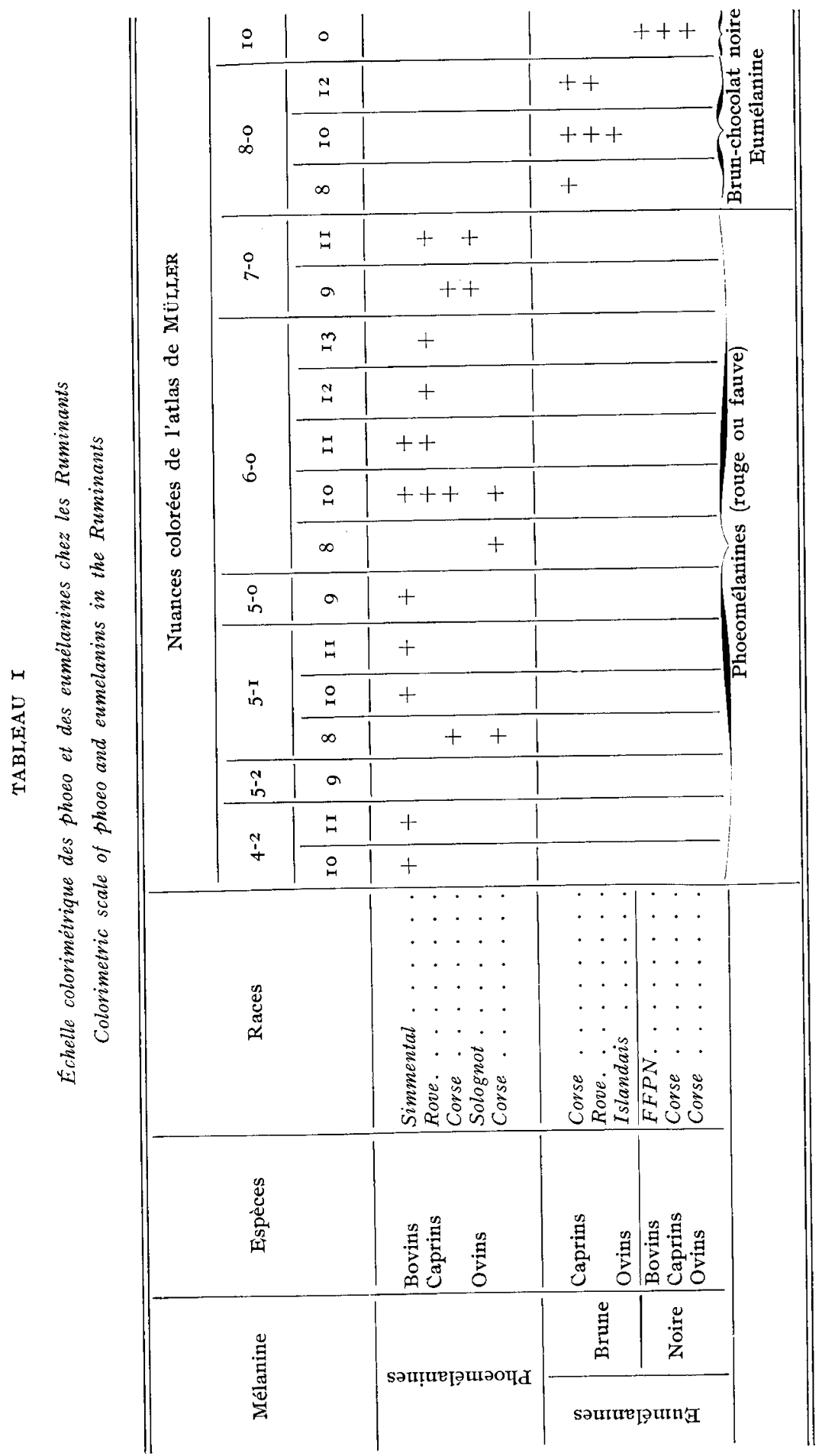


MOUFLON DE CORSE BRUN-CHOCOLAT

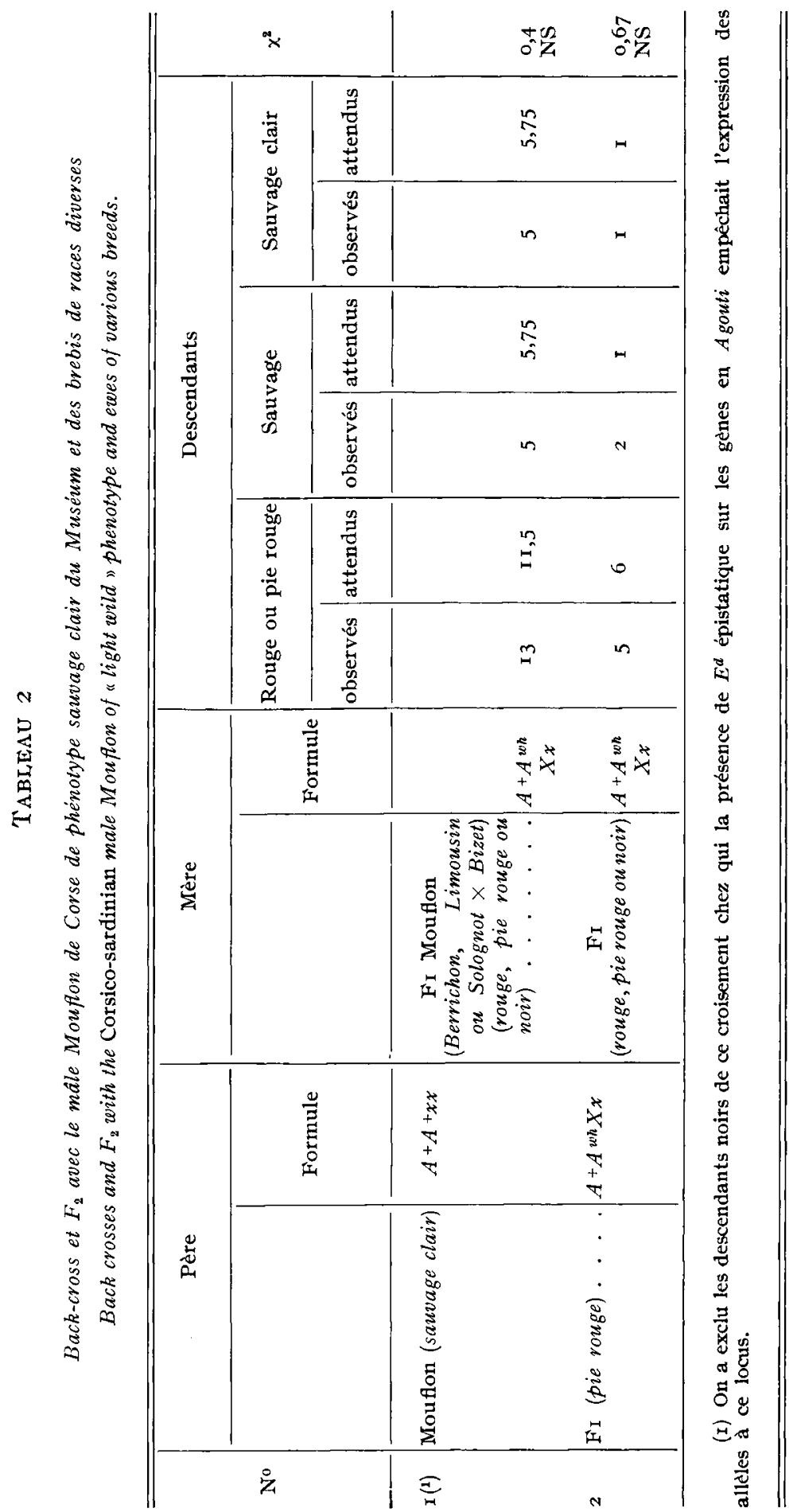


" valeur " qui permet de distinguer une couleur claire d'une couleur foncée et la " saturation " qui permet de différencier les couleurs ternes des couleurs vives (DÉRIBÉRÉ, I964). L'atlas MÜLLER que nous avons utilisé comporte 60 planches correspondant chacune à une teinte spectrale et numérotées de I à 60 . Il y a onze degrés de "valeur " (o à ro), et Io de saturation : o à 9. La désignation d'une observation se fait par sa teinte spectrale suivie du degré de valeur et saturation, par ex. : (IO) 7-2. L'atlas MÜILER comporte 254I nuances. Il s'inspire du système allemand D.I.N.

Pour obvier à la difficulté d'une représentation dans l'espace on a choisi, avec un certain arbitraire, une représentation dans le plan en tenant compte encore de la progression dans l'intensité des nuances.

\section{III. - Résultats}

\section{A. - Les ségrégations}

Des ségrégations clair/foncé au sein du type sauvage ont été observées dans les croisements de retour, et dans les $\mathrm{F}_{2}$ faits à Alfort à partir du Mouflon de Corse du Museum. Elles sont données dans le tableau 2 où l'on a, en outre, testé un dihybridisme : le mouflon étant $A^{+} A^{+} x x$, les $\mathrm{F}_{1}$ étant $A^{+} A^{w h} x X$ ( $A^{\text {wh }}$ est 1'allèle pour le rouge ou le blanc porté par les races parentales femelles Berrichon, Limousine ou Berrichon $\times$ Solognot, $A^{+}$est l'allèle sauvage pour le patron du mouflon, $x$ est l'allèle récessif modificateur responsable de l'éclaircissement).

\section{B. - Les types de mélanine dans le pelage des descendants de type "sauvage", clair ou non}

Chez les deux types "sauvage " et "sauvage clair ", la couleur fauve des flancs était (9) 5-2, (9) 7-0 ou (Ir) 7-0, ce qui correspond à des nuances de phoeomélanine des 3 espèces de Ruminants mesurées dans le tableau I.

La nuance des zones noires des phénotypes "sauvages " était ro-o et celle des zones foncées des " sauvage clair " (8) 8-0, cela correspond à des eumélanines noires dans le premier cas, brun-chocolat dans le second, selon le tableau I.

Fig. 2. - Les deux patrons colovés sauvage et sauvage clair.

a) Le mâle utilisé dans les $\mathrm{F}_{1}$ et back cross (de formule $A+A+b b$ ) sauvage clair.

b) Un produit de $2^{\mathrm{e}}$ génération également sauvage clair.

c) Femelle mouflon "sauvage " adulte $(A+A+B B)$ à droite, en fin de croissance de poil et jeune femelle sauvage clair à gauche $(A+A+b b)$ en mue (photo communiquée par J. ROUGEOT).

The two color patterns " wild " and "light wild"

a) The mouflon ram used in experimental crosses: light wild $A+A+b b$.

b) A lamb of the 2 nd generation cross also " light wild".

c) Adulte "wild" mouflon ewe $A+A+B B$ on the right (in hair growth phase) and a young mouflon female in moulting wearing the light wild pattern $A+A+b b$ (photograph by J. ROUGEOT). 

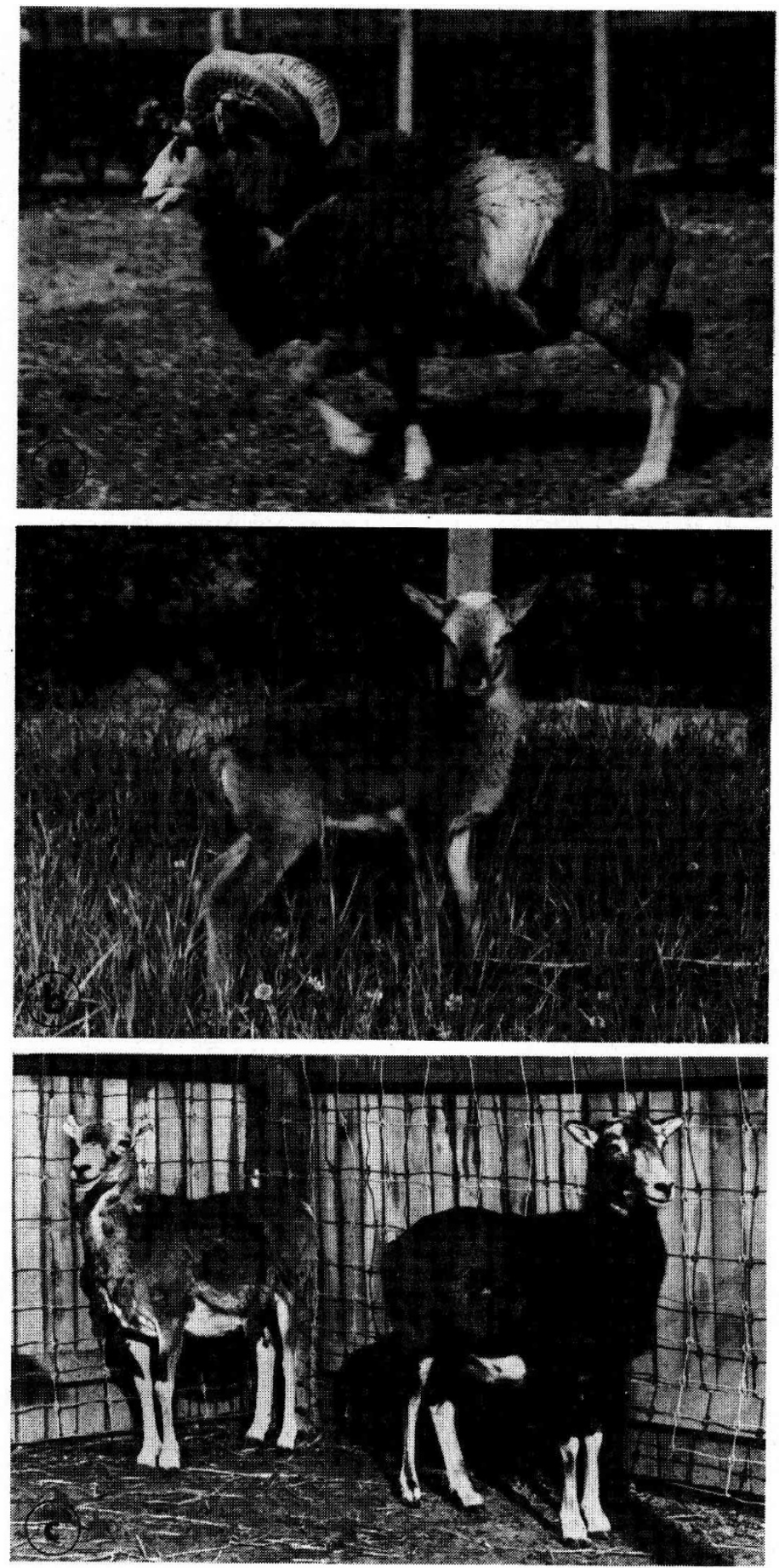


\section{Discussion}

La ségrégation d'un type clair parmi les individus de phénotype sauvage du tableau indique que l'on ne peut pas considérer que le sauvage clair est induit par un allèle en $A$ gouti.

Les résultats colorimétriques en I I B montrent que chez ces " sauvage clair » l'eumélanine noire semble bien avoir fait place à de l'eumélanine brun-chocolat.

Il semble donc que le variant " sauvage clair " soit dû̀ à allèle récessif au locus $B$ qui transforme l'eumélanine noire en eumélanine brun-chocolat. Cet allèle paraît, du moins dans sa manifestation phénotypique, (8) 8-o au lieu de (ro) 8-o, un peu différent de celui déjà isolé chez le mouton domestique et appelé $b$ par Adalsteinsson (I970). Avant de proposer un nouveau terme des mesures plus nombreuses semblent toutefois nécessaires. Il est à noter que, la couleur de l'eumélanine brune étant très proche de certaines nuances de la phoeomélanine, cela rendait difficile sa mise en évidence sans une échelle appropriée dans un pelage de type sauvage où les deux types de mélanines se retrouvent sur le même poil et où la phoeomélanine varie dans ses nuances (cf. fig. 2).

Il serait intéressant de savoir si le mutant existe parmi la population de Mouflons corses ou sardes vivant encore dans les îles, ce qui excluerait l'hypothèse d'une introduction lors de croisements intempestifs avec des ovins domestiques sur le continent avant la constitution de la souche du Museum.

Au cas où le mutant en $B$ appartiendrait en propre au patrimoine du Mouflon Corsico-Sarde, ce pourrait être un argument, venant après celui donné par 1'Hémoglobine A ( Bunch et al., I978) pour penser que le Mouflon de ces îles provient de la marronnisation d'un mouton domestique introduit il $\mathrm{y}$ a très longtemps (au $6^{\mathbf{e}}$ millénaire, alors que les débuts de la domestication au Moyen-Orient remontent au $8^{\mathrm{e}}$ millénaire av. J.-C.). Le mutant en $B$ serait alors celui du mouton archaïque. Dans une première étude sur les mutants colorés du mouton Corse indigène, LAUVERGNE et ADALSTEINSSON (I976) n'avaient toutefois pas trouvé d'allèles de ce type. Un examen plus récent et non encore publié semblerait pourtant prouver qu'il en existe. Cela demande encore confirmation.

Reçu pour publication en février 1979.

\section{Remerciements}

Les échantillons de laine brun-chocolat d'Islande ont été fournis par le $\mathrm{D}^{\mathrm{r}} \mathrm{S}$. ADALSTEINSSON, qui avait également suggéré une possible explication avec un mutant en $B$. Les mesures colorimétriques en race caprine du Rove ont été effectuées sur le troupeau de A. SADORGE.

\section{Summary}

$A$ light color variant due to an allele at the Brown $(B)$ locus in the Corsico-Sardinian Mouflon (Ovis Musimon)

A Corsico-sardinian male Mouflon of "light wild" phenotype which was used in France in a crossing experiment of coloration has been shown to carry a recessive gene at the color locus $B$ (Brown). This allele changes the color of eumelanin which, instead of being black, becomes chocolate-brown, close of some shades of red-yellow phoeomelanin. 


\section{Références bibliographiques}

AdAlsteinsson S., I970. Colour inheritance in Icelandic Sheep and relation between colour, fertility and fertilization. J. Agric. res. Icel., 2, 3-135.

Bunch T. D., N'Guyen T. C., Lauvergne J. J., 1978. Hemoglobitis of the Corsico-sardinian mouflon (Ovis musimon) and their implications for the origin of HbA in domestic sheep (Ovis avies). Ann. Génét. Sél. anim., 10, 503-506.

DERIBÉré M., I964. La couleur. P.U.F., Paris.

LaUvergne J. J., I966. Contribution à l'étude de l'hérédité de la couleur du pelage chez les bovins domestiques. Thèse Fac. Sci. de 1'Univ. de Paris ( $3^{\mathrm{e}}$ cycle).

Lauvergne J. J., Denis B., Théres M., I977. Hybridation entre un mouflon de Corse (Ovis ammon musimon, ScHREBER, I872) et des brebis de divers génotypes : gènes pour la coloration pigmentaire. Ann. Génét. Sél. anim., 9, 15I-16r.

LAUVERGNE J. J., HowEL, I, r978. Un premier inventaire génétique de la chèvre Corse (gènes à effets visibles). Ethnozootechnie, (22), 86-93.

MüLLER. Grösser mobiler schweizer Color Atlas 254r, Chromos Verlag, Winterthur Suisse.

SEARLE A. G., 1968. Comparative Genetics of Coat Colour in Mammals. Logos Press, Academic Press, London and New-York. 